\title{
Noncommutative Solitonic Black Hole
}

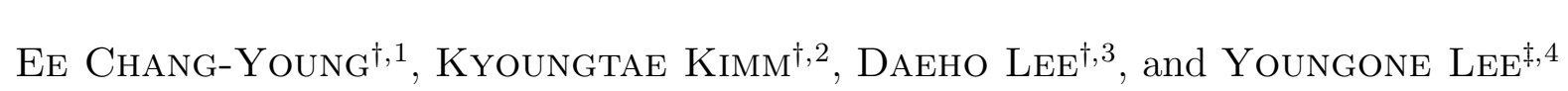 \\ ${ }^{\dagger}$ Department of Physics and Institute of Fundamental Physics, \\ Sejong University, Seoul 143-747, Korea \\ ${ }^{\ddagger}$ Institute of Basic Sciences, Daejin University, Pocheon, Gyeonggi 487-711, Korea
}

\begin{abstract}
We investigate solitonic black hole solutions in three dimensional noncommutative spacetime. We do this in gravity with negative cosmological constant coupled to a scalar field. Noncommutativity is realized with the Moyal product which is expanded up to first order in the noncommutativity parameter in two spatial directions. With numerical simulation we study the effect of noncommutativity by increasing the value of the noncommutativity parameter starting from commutative solutions. We find that even a regular soliton solution in the commutative case becomes a black hole solution when the noncommutativity parameter reaches a certain value.
\end{abstract}

\footnotetext{
${ }^{1}$ cylee@sejong.ac.kr

${ }^{2}$ ktk@theory.sejong.ac.kr

${ }^{3}$ dhlee@theory.sejong.ac.kr

4youngone@daejin.ac.kr
} 
Many candidate theories for quantum gravity, such as string theory and loop quantum gravity, suggest that spacetime may not be commutative at sufficiently high energy scales [1, 2]. Meanwhile, black holes in the early universe have been observed recently [3, 4]. Since the energy density of the early universe was very high, it would be interesting to know the effect of noncommutativity on the formation of a black hole. Black holes in three dimensional spacetime have been extensively studied. One of the reasons is that gravity models in three dimensions are relatively easier to treat than models in four spacetime dimensions. The finding of the BTZ black hole solution [5] has raised a lot of interest in the subject.

In [6], the global vortex solution was studied by considering gravity with negative cosmological constant coupled to a complex scalar field in three dimensional commutative spacetime. There, the model Lagrangian with a global $U(1)$ symmetry was given by

$$
S=\int d^{3} x \sqrt{-g}\left[-\frac{1}{16 \pi G_{N}}(R+2 \Lambda)+\frac{1}{2} g^{\mu \nu} \partial_{\mu} \bar{\phi} \partial_{\nu} \phi-\frac{\lambda}{4}\left(\bar{\phi} \phi-v^{2}\right)^{2}\right],
$$

where $\phi(x)$ is a complex scalar field. The obtained solution was a cylindrically symmetric global $\mathrm{U}(1)$ vortex solution which smoothly connects the false vacuum at the origin to the true vacuum at spatial infinity. Depending upon the ratio of the cosmological constant to the Plank scale, the model supports a spacetime with a regular soliton or a charged black hole.

In this paper, we investigate the effect of noncommutativity on the above model and want to see whether global vortex solutions with black hole configuration are allowed in the noncommutative case. For this purpose we use the same Lagrangian as in (11) except for the Moyal product between the field variables. For computational purpose, here we use the triad and spin connection instead of the metric as in [7]. Since it is hard to obtain an analytic solution even in the commutative case [6], our approach to find the solution is basically numerical. Our analysis is performed up to first order in the noncommutativity parameter.

In this paper we work with the noncommutative polar coordinates $(\hat{t}, \hat{r}, \hat{\varphi})$ defined by the following commutation relation

$$
[\hat{\rho}, \hat{\varphi}]=2 i \theta, \quad 0 \text { otherwise }
$$


where $\hat{\rho} \equiv \hat{r}^{2}$. One reason for using the above commutation relation is that rotational symmetry is more apparent in the polar coordinates than in the Cartesian coordinates. Another quite important reason is that the above commutation relation is physically equivalent to that of the canonical noncommutivity, $[\hat{x}, \hat{y}]=i \theta$, up to first order in the nonocmmutativity parameter $\theta$ [7]. A would-be usual commutation relation for the noncommutative polar coordinates, $[\hat{r}, \hat{\varphi}]=i \theta$, is not equivalent to the canonical noncommutivity, $[\hat{x}, \hat{y}]=i \theta$, even in the first order of $\theta[7]$.

By the Weyl-Moyal correspondence [8], the physics in the noncommutative spacetime can be described by the physics in the commutative spacetime with the Moyal product. The Moyal product corresponding to the commutation relation (2) is given by

$$
(f \star g)(\rho, \varphi)=\left.e^{i \theta\left(\frac{\partial}{\partial \rho} \frac{\partial}{\partial \varphi^{\prime}}-\frac{\partial}{\partial \varphi} \frac{\partial}{\partial \rho^{\prime}}\right)} f(\rho, \varphi) g\left(\rho^{\prime}, \varphi^{\prime}\right)\right|_{(\rho, \varphi)=\left(\rho^{\prime}, \varphi^{\prime}\right)} .
$$

Since the pure gravity action with negative cosmological constant can be written as the Chern-Simons action [9], the first two terms of the action (11) can be rewritten in terms of the triad $e^{a}$ and the spin connection $\omega^{a}(a=0,1,2)$. Then the noncommutative version of the action (1) can be written as

$$
\hat{S}=\frac{1}{8 \pi G_{N}} \int\left(\hat{e}_{a} \wedge^{\star} \hat{R}^{a}+\frac{\Lambda}{6} \epsilon^{a b c} \hat{e}_{a} \wedge^{\star} \hat{e}_{b} \wedge^{\star} \hat{e}_{c}\right)+\int d^{3} x \hat{e} \star \hat{\mathcal{L}}[\hat{\phi}]
$$

where $\hat{e}$ is the determinant of $\hat{e}_{\mu}^{a}$ and $\hat{R}^{a}$ is the curvature 2 -form, $\hat{R}^{a}=d \hat{\omega}^{a}+\frac{1}{2} \epsilon^{a b c} \hat{\omega}_{b}{ }^{\star} \hat{\omega}_{c}$, and the Lagrangian $\hat{\mathcal{L}}[\hat{\phi}]$ for the scalar field is given by

$$
\hat{\mathcal{L}}[\hat{\phi}]=-\frac{1}{4}\left(\partial_{\mu} \hat{\bar{\phi}} \star g^{\mu \nu} \star \partial_{\nu} \hat{\phi}+\partial_{\mu} \hat{\phi} \star g^{\mu \nu} \star \partial_{\nu} \hat{\bar{\phi}}\right)-\frac{\lambda}{4}\left(\hat{\bar{\phi}} \star \hat{\phi}-v^{2}\right) \star\left(\hat{\bar{\phi}} \star \hat{\phi}-v^{2}\right) .
$$

We define the noncommutative metric as

$$
\hat{g}_{\mu \nu}=\frac{1}{2} \eta_{a b}\left(\hat{e}_{\mu}^{a} \star \hat{e}_{\nu}^{b}+\hat{e}_{\nu}^{b} \star \hat{e}_{\mu}^{a}\right)
$$


such that it is real and symmetric. The equations of motion are as follows:

$$
\begin{aligned}
& \frac{\epsilon^{\mu \nu \rho}}{8 \pi G_{N}}\left[\hat{R}_{a}+\frac{\Lambda}{2} \epsilon_{a b c} \hat{e}^{b} \stackrel{\wedge}{\hat{e}}^{c}\right]_{\nu \rho}+\frac{1}{6} \epsilon^{\mu \nu \rho} \epsilon_{a b c}\left(\hat{e}_{\nu}^{b} \star \hat{e}_{\rho}^{c} \star \hat{\mathcal{L}}[\hat{\phi}]+\hat{e}_{\rho}^{c} \star \hat{\mathcal{L}}[\hat{\phi}] \star \hat{e}_{\nu}^{b}+\hat{\mathcal{L}}[\hat{\phi}] \star \hat{e}_{\nu}^{b} \star \hat{e}_{\mu}^{b}\right) \\
& +\frac{1}{8}\left[\hat{e}_{c}^{\mu} \star \hat{e}_{b}^{\nu} \star \partial_{\nu} \hat{\phi} \star \hat{e} \star \partial_{\rho} \hat{\bar{\phi}} \star \hat{e}_{a}^{\rho}+\hat{e}_{c}^{\mu} \star \partial_{\nu} \hat{\phi} \star \hat{e} \star \partial_{\rho} \hat{\bar{\phi}} \star \hat{e}_{b}^{\nu} \star \hat{e}_{a}^{\rho}\right. \\
& \left.+\hat{e}_{b}^{\mu} \star \partial_{\nu} \hat{\phi} \star \hat{e} \star \partial_{\rho} \hat{\bar{\phi}} \star \hat{e}_{c}^{\rho} \star \hat{e}_{a}^{\nu}+\hat{e}_{b}^{\mu} \star \hat{e}_{c}^{\rho} \star \partial_{\nu} \hat{\phi} \star \hat{e} \star \partial_{\rho} \hat{\bar{\phi}} \star \hat{e}_{a}^{\nu}+(\hat{\phi} \leftrightarrow \hat{\bar{\phi}})\right]=0 \\
& \hat{T}^{a} \equiv d \hat{e}^{a}+\frac{1}{2} \epsilon^{a b c}\left(\hat{\omega}_{b} \stackrel{\wedge}{\wedge}^{\hat{e}_{c}}+\hat{e}_{b}{ }^{\star} \hat{\omega}_{c}\right)=0 \\
& \partial_{\mu}\left(\hat{g}^{\mu \nu} \star \partial_{\nu} \hat{\phi} \star \hat{e}+\hat{e} \star \partial_{\nu} \hat{\phi} \star \hat{g}^{\mu \nu}\right)=\lambda \hat{\phi} \star\left(\hat{\bar{\phi}} \star \hat{\phi} \star \hat{e}+\hat{e} \star \hat{\bar{\phi}} \star \hat{\phi}-2 v^{2} \hat{e}\right)
\end{aligned}
$$

In the commutative limit, $\theta \rightarrow 0$, the equations (17)-(9) reduce to the commutative ones [10, 6] as expected.

In the commutative case, a static and rotationally symmetric metric can be put into the following form [6]:

$$
d s^{2}=-e^{2 A(r)} B(r) d t^{2}+\frac{d r^{2}}{B(r)}+r^{2} d \varphi^{2} .
$$

The triad and spin connection corresponding to this line element are given by [10]

$$
\begin{aligned}
& e^{0}=e^{A} \sqrt{B} d t, \quad e^{1}=\frac{1}{\sqrt{B}} d r, \quad e^{2}=r d \varphi \\
& \omega^{0}=-\sqrt{B} d \varphi, \quad \omega^{1}=0, \quad \omega^{2}=-\sqrt{B} \frac{d}{d r}\left(e^{A} \sqrt{B}\right) d t .
\end{aligned}
$$

In terms of $A, B$, and $\phi$, where $\phi \equiv|\phi|(r) e^{i n \varphi}$, the commutative equations of motion reduced from (7)-(9) are given by

$$
\begin{aligned}
& A^{\prime}=8 \pi G_{N} r\left(|\phi|^{\prime}\right)^{2}, \\
& B^{\prime}=2|\Lambda| r-8 \pi G_{N} r\left[B\left(|\phi|^{\prime}\right)^{2}+\frac{n^{2}}{r^{2}}|\phi|^{2}+\frac{\lambda}{2}\left(|\phi|^{2}-v^{2}\right)^{2}\right], \\
& |\phi|^{\prime \prime}+\left(A^{\prime}+\frac{B^{\prime}}{B}+\frac{1}{r}\right)|\phi|^{\prime}=\frac{1}{B}\left(\frac{n^{2}}{r^{2}}+\lambda\left(|\phi|^{2}-v^{2}\right)\right)|\phi|,
\end{aligned}
$$

and these are exactly the same equations that appeared in [6].

In line with the Weyl-Moyal correspondence and taking a hint from the commutative metric (10), we now make an ansatz for the noncommutative metric in the $(t, \rho, \varphi)$ coordinates 


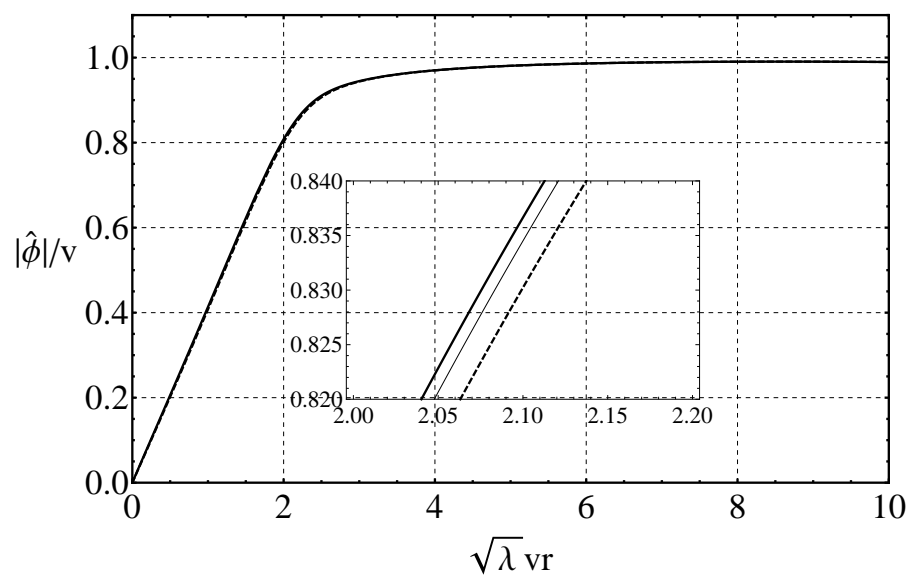

Figure 1: The noncommutative vortex solutions with $\Lambda_{v}=0.1$ and $G_{v}=1.33$ are plotted for the scalar field $|\hat{\phi}| / v$. The dashed line is for the case of $\theta=0$. The thin and thick solid lines are for the cases of $\theta=0.1,0.15$, respectively.

which satisfy the commutation relation (2) as follows:

$$
d \hat{s}^{2}=-e^{2 \hat{A}(\rho)} \hat{B}(\rho) d t^{2}+\frac{d \rho^{2}}{4 \rho \hat{B}(\rho)}+\rho d \varphi^{2} .
$$

A noncommutative triad for the above metric compatible with the definition of the metric (6) can be chosen as

$$
\hat{e}^{0}=e^{\hat{A}(\rho)} \sqrt{\hat{B}(\rho)} d t, \quad \hat{e}^{1}=\frac{d \rho}{\sqrt{4 \rho \hat{B}(\rho)}}, \hat{e}^{2}=\sqrt{\rho} d \varphi .
$$

With the above choice of the triad, the noncommutative spin connection can be determined from the noncommutative torsion free condition (8):

$$
\hat{\omega}^{0}=-\sqrt{\hat{B}(\rho)} d \varphi, \hat{\omega}^{1}=0, \hat{\omega}^{2}=-\sqrt{4 \rho \hat{B}(\rho)} \frac{d}{d \rho}\left(e^{\hat{A}(\rho)} \sqrt{\hat{B}(\rho)}\right) d t
$$

Now we expand the metric functions $\hat{A}, \hat{B}$, and the scalar field $\hat{\phi}$ for static global vortices with vorticity $n$ in terms of $\theta$ up to first order as follows.

$$
\begin{aligned}
e^{2 \hat{A}(\rho)} \hat{B}(\rho) & =e^{2 \tilde{A}(\rho)} \tilde{B}(\rho)+\theta \tilde{F}(\rho)+\mathcal{O}\left(\theta^{2}\right), \\
\hat{B}(\rho) & =\tilde{B}(\rho)+\theta \tilde{G}(\rho)+\mathcal{O}\left(\theta^{2}\right), \\
\hat{\phi}(\rho, \varphi) & =(\tilde{\phi}(\rho)+\theta \tilde{\Phi}(\rho)) e^{i n \varphi}+\mathcal{O}\left(\theta^{2}\right) .
\end{aligned}
$$


With the following redefinitions of functions,

$$
\begin{gathered}
\tilde{A}(\rho) \equiv A(r), \tilde{B}(\rho) \equiv B(r), \tilde{\phi}(\rho) \equiv|\phi|(r), \\
\tilde{F}(\rho) \equiv F(r), \tilde{G}(\rho) \equiv G(r), \tilde{\Phi}(\rho) \equiv \Phi(r),
\end{gathered}
$$

and from (7)-(9), we get the original commutative equations (11) in the zeroth order of $\theta$, and the following three equations for $F(r), G(r), \Phi(r)$ in the first order of $\theta$ :

$$
\begin{aligned}
& a_{1}(r) F(r)+a_{2}(r) F^{\prime}(r)+a_{3}(r) G(r)+a_{4}(r) G^{\prime}(r) \\
& +a_{5}(r) \Phi(r)+a_{6}(r) \Phi^{\prime}(r)+a_{7}(r) \Phi^{\prime \prime}(r)+a_{8}(r)=0, \\
& b_{1}(r) G(r)+b_{2}(r) G^{\prime}(r)+b_{3}(r) \Phi(r)+b_{4}(r) \Phi^{\prime}(r)+b_{5}(r)=0, \\
& c_{1}(r) F(r)+c_{2}(r) F^{\prime}(r)+c_{3}(r) G(r)+c_{4}(r) \Phi(r)+c_{5}(r) \Phi^{\prime}(r)+c_{6}(r)=0 .
\end{aligned}
$$

All the coefficients in these equations are functions of the known commutative solution, $|\phi|$, $A$ and $B$, and are given in the appendix.

The numerical analysis was performed up to first order in the noncommutativity parameter $\theta$ for the vorticity $n=1$. The cosmological constant, the Newton constant, and the radial coordinate are scaled to $\Lambda_{v}=|\Lambda| / \lambda v^{2}, G_{v}=8 \pi G_{N} v^{2}$, and $\sqrt{\lambda} v r$, respectively. In order to solve (15) we impose the Dirichlet condition at the origin for $F$ and $G$ which is

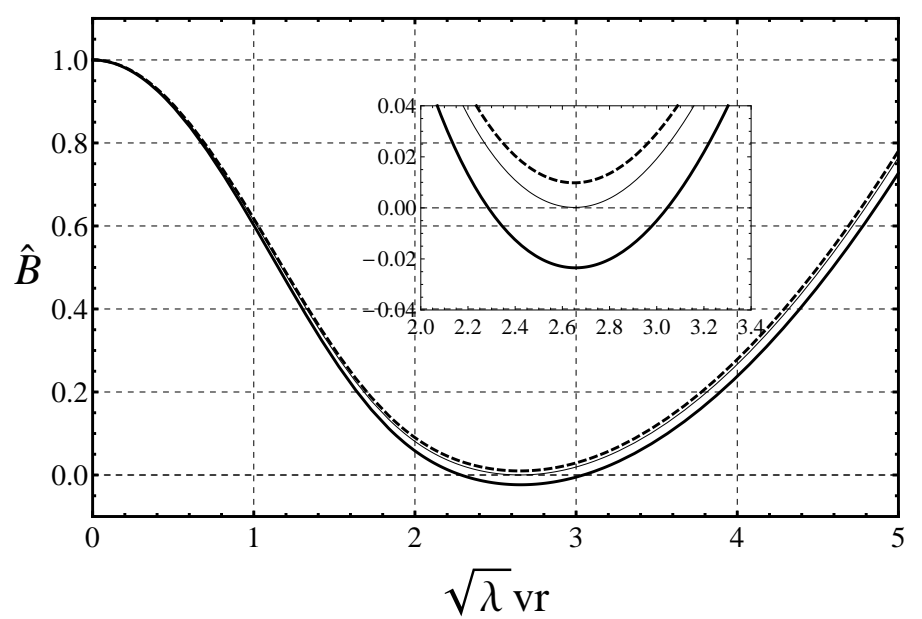

Figure 2: The solutions for noncommutative metric are plotted for $\hat{B}$ at values $\Lambda_{v}=0.088$ and $G_{v}=1.33$. The dashed line is for the case of $\theta=0$. The thin and thick solid lines are for the cases of $\theta=0.057,0.2$, respectively. We note that there is a fine split at $r=0$. 


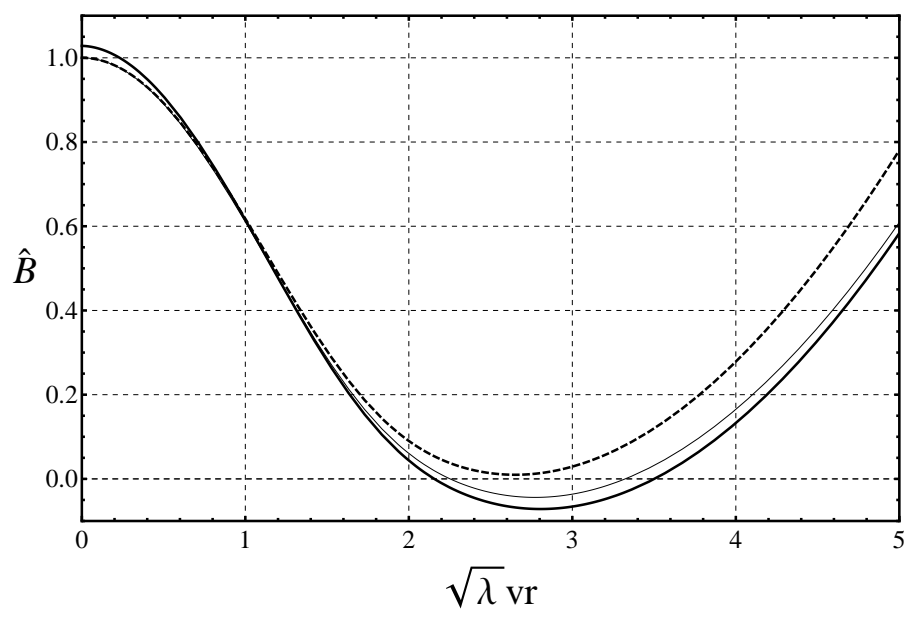

Figure 3: The noncommutative black hole solutions with $G_{v}=1.33$ are plotted for $\hat{B}$. The dashed and thin solid lines are for the cases of $\Lambda_{v}=0.087,0.08$, respectively, at $\theta=0$. The thick solid line is for the case of $\Lambda_{v}=0.08$ at $\theta=0.2$.

compatible with the boundary condition for $\Phi(r)$

$$
\Phi(0)=\Phi(\infty)=0
$$

The effects of turning on $\theta$ on the global vortex solutions is shown in Fig. 1. This shows that the scalar field concentrates inwards as $\theta$ grows. The gradient of the scalar field around $r=0$ becomes steeper. In Fig. 2, the metric function $\hat{B}$ corresponding to the global vortices in Fig. 1 is drawn. Note that the commutative solution $(\theta=0)$ has no horizon. On the other hand, when the value of the noncommutativity parameter $\theta$ reaches 0.057 , the solution becomes an extremal black hole solution. When the value of $\theta$ becomes larger than this value, for instance $\theta=0.2$, it becomes a nonextremal black hole solution. When the starting commutative solution is a nonextremal black hole solution with $\Lambda_{v}=0.08$, the corresponding noncommutative solution for $\theta=0.2$ is shown in Fig. 3. The effect of turning on $\theta$ for alreadyblack holes in commutative spacetime is shown. As the noncommutativity parameter $\theta$ gets bigger, the area of the outer horizon increases while the separation between the inner and outer horizons grows. This effect is what we would expect when the mass of a nonextremal black hole increases in the commutative case.

In order to see whether the singularities of the metric at zeros are coordinate artifacts or true physical singularities, the Kretschmann scalars $\hat{R}_{\mu \nu \rho \sigma} \hat{R}^{\mu \nu \rho \sigma}$ for the solutions having 


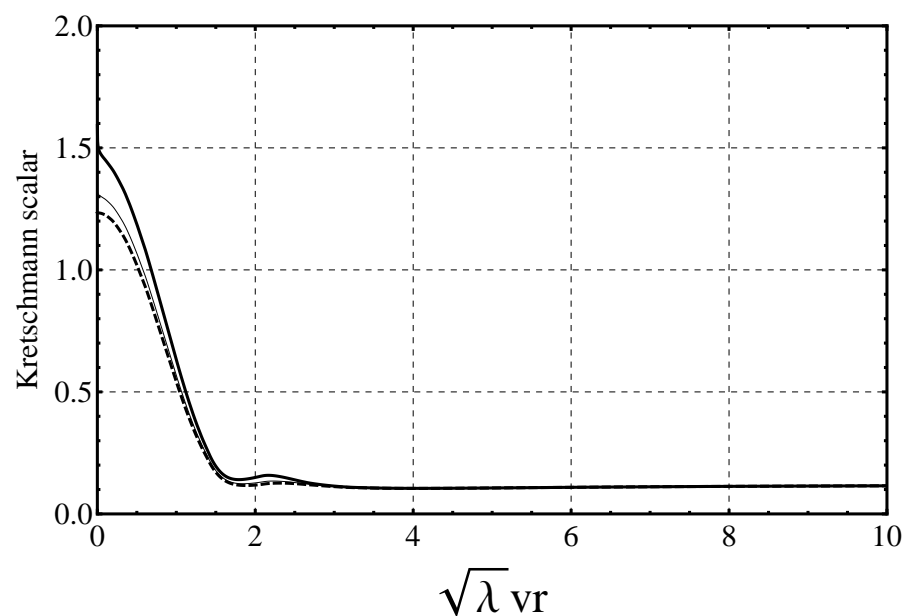

Figure 4: The plot of the noncommutative Kretschmann scalar with $\Lambda_{v}=0.1$ and $G_{v}=1.33$. The dashed line is for the case of $\theta=0$. The thin and thick solid lines are for the cases of $\theta=0.057,0.2$, respectively.

one or two zeros in Fig. 2 are plotted in Fig. 4, This shows that the Kretschmann invariant for the regions around these zeros behaves regularly. From this result we can say that the zeros are not genuine singularities, rather they correspond to the horizons of black holes.

The increase in gravitational mass for different values of $\theta$ by using the Hamiltonian formalism of [11] is plotted in Fig. [5. The increase in gravitational mass for a given $\theta$ is defined by

$$
\Delta M \equiv H(\theta \neq 0)-H(\theta=0)
$$

where $H$ denotes the Hamlitonian. The result shows that the gravitational mass increases as the noncomutativity parameter $\theta$ increases. This linearity shown in Fig. 5 is due to our analysis which was performed up to first order in $\theta$. The gravitational mass defined here depends only on the asymptotic geometrical quantities at spatial infinity. The metric is fully determined by the scalar field and its derivatives which are constant at spatial infinity. Since the coefficient of $\theta$ is constant, it implies the linear dependence of mass on $\theta$.

Thus our result sums up as follows. The 'inward' behaviour of the global vortex in Fig. 1 results in higher peaks of the Kretschmann scalar near $r=0$ as in Fig. 4. These peaks correspond to gravitational energy concentrations. We may interpret this as the noncommutativity of space makes the scalar soliton have higher concentration near the center than in the commutative case. This concentrated scalar soliton behaves as the concentration 


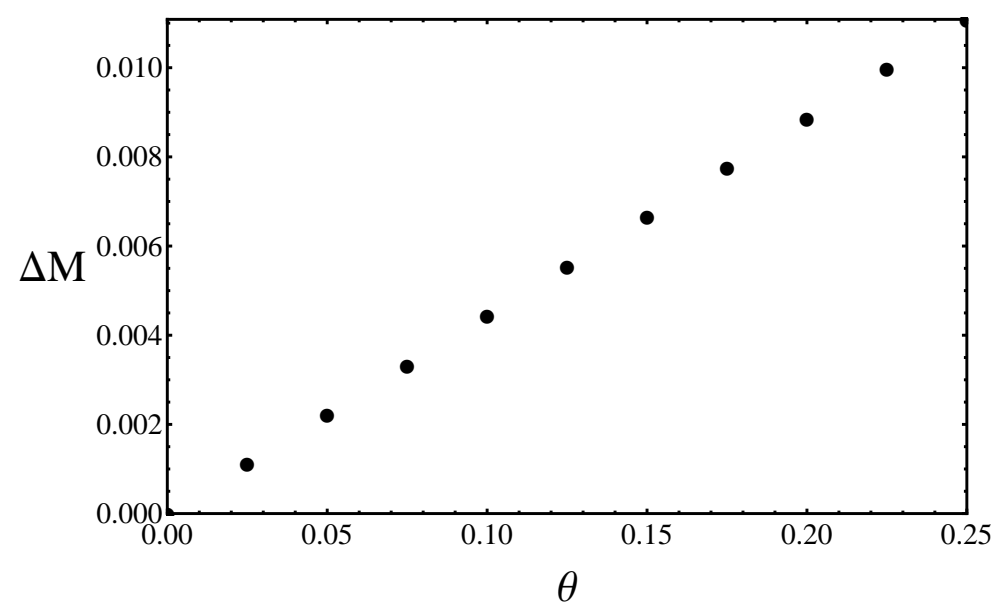

Figure 5: The plot of the gravitational mass increase vs $\theta$ with $\Lambda_{v}=0.1$ and $G_{v}=1.33$.

of gravitational energy to such an extent that a black hole can form.

In the space of solutions, a solution with no singularity changes into a black hole solution as $\theta$ increases. In other words, as one increases the value of the noncommutativity parameter, the spacetime starts to allow a black hole solution at a certain value of the noncommutativity parameter. This is not what we expected before. A 'phase change' in the solution space happens as the noncommutativity parameter changes.

Comparing with the result obtained in [7] for noncommutative BTZ black hole, the role of the scalar field in the present work on forming a black hole is similar to that of the magnetic flux $B$ there. The shift of the locations of the horizons of the noncommutative black holes in that paper depends upon both $\theta$ and the magnetic flux $B$ at the origin. The shift of horizons obtained in this work depends on both $\theta$ and the scalar field. Thus, one may infer that the global vortex around the origin takes over the role of the magnetic flux in [7].

\section{Acknowledgments}

This work was supported by the National Research Foundation (NRF) of Korea grants funded by the Korean government (MEST) [R01-2008-000-21026-0 and NRF-2009-0075129 (E. C.-Y., K. K. and D. L.), and The Korea Research Foundation Grant funded by the Korea Government(MOEHRD), KRF-2008-314-C00063(Y.L.) 


\section{Appendix}

The coefficients appearing in the equations (15) for $F(r), G(r), \Phi(r)$ are given as follows:

$$
\begin{aligned}
& a_{1}(r)=-r\left[\left(n^{2}-\lambda v^{2} r^{2}\right) \phi+\lambda r^{2} \phi^{3}-r B\left(\left(1-r A^{\prime}\right) \phi^{\prime}+r \phi^{\prime \prime}\right)\right], \\
& a_{2}(r)=r^{3} B \phi^{\prime}, \\
& a_{3}(r)=r e^{2 A}\left[\left(n^{2}-\lambda v^{2} r^{2}\right) \phi+\lambda r^{2} \phi^{3}+r B\left(\left(1+r A^{\prime}\right) \phi^{\prime}+r \phi^{\prime \prime}\right)\right], \\
& a_{4}(r)=e^{2 A} r^{3} B \phi^{\prime}, \\
& a_{5}(r)=-2 e^{2 A} r B\left(n^{2}-\lambda v^{2} r^{2}+3 \lambda r^{2} \phi^{2}\right), \\
& a_{6}(r)=2 e^{2 A} r^{2} B\left(B+r B A^{\prime}+r B^{\prime}\right), \\
& a_{7}(r)=2 e^{2 A} r^{3} B^{2}, \\
& a_{8}(r)=n B e^{2 A}\left[\left(n^{2}+2 \lambda v^{2} r^{2}\right) \phi A^{\prime}-2 \lambda r^{2} \phi^{3} A^{\prime}-r^{2}\left(A^{\prime} B^{\prime} \phi^{\prime}+B\left(A^{\prime 2} \phi^{\prime}+\phi^{\prime} A^{\prime \prime}+A^{\prime} \phi^{\prime \prime}\right)\right)\right] \\
& b_{1}(r)=\frac{1}{2}\left[r\left(B^{\prime}-2 \Lambda r\right)+4 \pi G_{N}\left(2\left(n^{2}-\lambda v^{2} r^{2}\right) \phi^{2}+\lambda r^{2} \phi^{4}+r^{2}\left(\lambda v^{4}-2 B \phi^{2}\right)\right)\right], \\
& b_{2}(r)=-r B, \\
& b_{3}(r)=-16 \pi G_{N} B \phi\left(n^{2}-\lambda v^{2} r^{2}+\lambda r^{2} \phi^{2}\right), \\
& b_{4}(r)=-16 \pi G_{N} r^{2} B^{2} \phi^{\prime}, \\
& b_{5}(r)=8 \pi G_{N} n \lambda r B \phi\left(\phi^{2}-v^{2}\right) \phi^{\prime}, \\
& c_{1}(r)=\frac{1}{2}\left[r\left(2 \Lambda r+2 B A^{\prime}+B^{\prime}\right)-4 \pi G_{N}\left(2\left(n^{2}-\lambda v^{2} r^{2}\right) \phi^{2}+\lambda r^{2} \phi^{4}+r^{2}\left(\lambda v^{4}-2 B \phi^{\prime 2}\right)\right)\right], \\
& c_{2}(r)=-r B \\
& c_{3}(r)=-r e^{2 A}\left(B^{\prime}+2 B\left(A^{\prime}-4 \pi G_{N} r \phi^{\prime 2}\right)\right), \\
& c_{4}(r)=-16 \pi G_{N} e^{2 A} B \phi\left(n^{2}-\lambda v^{2} r^{2}+\lambda r^{2} \phi^{2}\right), \\
& c_{5}(r)=16 \pi G_{N} e^{2 A} r^{2} B^{2} \phi^{\prime}, \\
& c_{6}(r)=8 \pi G_{N} e^{2 A} n \lambda r B \phi\left(\phi^{2}-v^{2}\right) \phi^{\prime} . \\
& =
\end{aligned}
$$

\section{References}

[1] N. Seiberg and E. Witten, String Theory and Noncommutative Geometry, JHEP 09 (1999) 032 [hep-th/9908142]. 
[2] L. Freidel and E. R. Livine, 3D Quantum Gravity and Effective Noncommutative Quantum Field Theory, Phys. Rev. Lett. 96 (2006) 221301 hep-th/0512113.

[3] X. Fan et al., High-Redshift Quasars Found in Sloan Digital Sky Survey Commissioning Data IV: Luminosity Function from the Fall Equatorial Stripe Sample, Astron. J. 121 (2001) 54 astro-ph/0008123.

[4] C. J. Willott et al., Four quasars above redshift 6 discovered by the Canada-France High-z Quasar Survey, Astron. J. 134 (2007) 2435 arXiv:0706.0914.

[5] M. Banados, C. Teitelboim, and J. Zanelli, The Black Hole in Three Dimensional Space Time, Phys. Rev. Lett. 69 (1992) 1849 [hep-th/9204099].

[6] N. Kim, Y. Kim, and K. Kimm, Global Vortex and Black Cosmic String, Phys. Rev. D 56 (1997) 8029 [gr-qc/9707056].

[7] Ee C.-Y., D. Lee, and Y. Lee, Noncommutative BTZ Black Hole in Polar Coordinates, Class. Quant. Grav. 26 (2009) 185001 arXiv:0808.2330.

[8] H. J. Groenewold, On the Principles of elementary quantum mechanics, Physica 12 (1946) 405; J. E. Moyal, Quantum mechanics as a statistical theory, Proc. Cambridge Phil. Soc. 45 (1949) 99.

[9] A. Achucarro and P. K. Townsend, A Chern-Simons Action for Three-Dimensional anti-De Sitter Supergravity Theories, Phys. Lett. B 180 (1986) 89; E. Witten, (2+1)Dimensional Gravity as an Exactly Soluble System, Nucl. Phys. B 311 (1988) 46.

[10] S. Carlip, J. Gegenberg, and R. B. Mann, Black Holes in Three Dimensional Topological Gravity, Phys. Rev. D 51 (1995) 6854 gr-qc/9410021.

[11] S. W. Hawking and G. T. Horowitz, The Gravitational Hamiltonian, Action, Entropy, and Surface Terms, Class. Quant. Grav. 13 (1996) 1487 [gr-qc/9501014]. 